A peer-reviewed version of this preprint was published in PeerJ on 1 March 2018.

View the peer-reviewed version (peerj.com/articles/4449), which is the preferred citable publication unless you specifically need to cite this preprint.

Brüwer JD, Voolstra CR. 2018. First insight into the viral community of the cnidarian model metaorganism Aiptasia using RNA-Seq data. Peerj 6:e4449 https://doi.org/10.7717/peerj.4449 


\section{Insights into viral community composition of the cnidarian model metaorganism Aiptasia using RNA-Seq data}

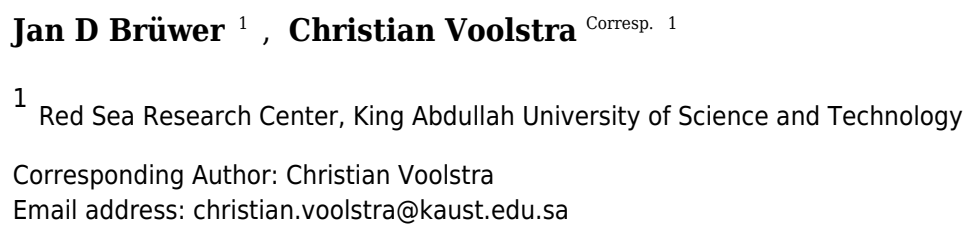

Current research posits that all multicellular organisms live in symbioses with associated microorganisms and form so-called metaorganisms or holobionts. Cnidarian metaorganisms are of specific interest given that stony corals provide the foundation of the globally threatened coral reef ecosystems and their well-being strongly relies on forming mutualistic relationships with endosymbiotic algae of the genus Symbiodinium. So far, only few studies characterized viral diversity and the potential underlying functional importance to coral holobionts. Here we analyzed an existing RNA-Seq dataset of the coral model metaorganism Aiptasia CC7 (sensu Exaiptasia pallida) associated with aposymbiotic, partially populated, and fully symbiotic anemones with Symbiodinium to gain further insight into viral community composition and the relation to the algal endosymbiosis. Our approach included the selective removal of anemone host and algal endosymbiont sequences and subsequent microbial sequence annotation. Of a total of 297 million raw sequence reads, 8.6 million $(\sim 3 \%)$ remained after host and endosymbiont sequence removal. Of these, 3,293 sequences (paired-end read pairs) could be assigned as of viral origin. Taxonomic annotation shows that Aiptasia is associated with a diverse viral community consisting of 116 viral taxa covering 40 families. The viral community was dominated by viruses from the families Herpesviridae (12.00\%), Partitiviridae (9.93\%), and Picornaviridae (9.87\%). Despite an overall stable viral community, we found that some viral taxa significantly changed in relative abundance when Aiptasia engage in a symbiotic relationship with Symbiodinium. Elucidation of viral taxa consistently present in all samples revealed an Aiptasia core virome of 15 viral taxa from 11 viral families that was comprised of many viruses previously reported in coral viromes. Our study provides a first insight into the viral community of Aiptasia. Aiptasia seem to harbor a diverse and overall stable viral community, although certain members change in abundance when the anemone host associates with its algal endosymbiont. However, the functional significance of this remains to be determined. 
1 Insights into viral community composition of the cnidarian model metaorganism Aiptasia

2 using RNA-Seq data

3

4 Jan D Brüwer ${ }^{1}$, Christian R Voolstra ${ }^{1^{*}}$

5

6 Author Affiliations

$7 \quad{ }^{1}$ Red Sea Research Center, Division of Biological and Environmental Science and Engineering

8 (BESE), King Abdullah University of Science and Technology (KAUST), Thuwal 23955-6900, Saudi

9 Arabia

10

$11 *$ Corresponding Author

12 Email: Christian.voolstra@kaust.edu.sa

13 Phone: +966 544700087

14 Fax: +966 218082377

15

16 Short title

17 Viral community of Aiptasia CC7

18

19 Keywords

20 Model organism, Aiptasia, Exaiptasia pallida, virus, RNA-Seq, metaorganism, holobiont,

21 symbiosis

22 


\section{Abstract}

24 Current research posits that all multicellular organisms live in symbioses with associated 25 microorganisms and form so-called metaorganisms or holobionts. Cnidarian metaorganisms are 26 of specific interest given that stony corals provide the foundation of the globally threatened 27 coral reef ecosystems and their well-being strongly relies on forming mutualistic relationships with endosymbiotic algae of the genus Symbiodinium. So far, only few studies characterized viral diversity and the potential underlying functional importance to coral holobionts. Here we analyzed an existing RNA-Seq dataset of the coral model metaorganism Aiptasia CC7 (sensu Exaiptasia pallida) associated with aposymbiotic, partially populated, and fully symbiotic anemones with Symbiodinium to gain further insight into viral community composition and the relation to the algal endosymbiosis. Our approach included the selective removal of anemone host and algal endosymbiont sequences and subsequent microbial sequence annotation. Of a 35 total of 297 million raw sequence reads, 8.6 million ( 3\%) remained after host and endosymbiont sequence removal. Of these, 3,293 sequences (paired-end read pairs) could be assigned as of viral origin. Taxonomic annotation shows that Aiptasia is associated with a diverse viral community consisting of 116 viral taxa covering 40 families. The viral community was dominated by viruses from the families Herpesviridae (12.00\%), Partitiviridae (9.93\%), and Picornaviridae (9.87\%). Despite an overall stable viral community, we found that some viral taxa significantly changed in relative abundance when Aiptasia engage in a symbiotic relationship with Symbiodinium. Elucidation of viral taxa consistently present in all samples revealed an Aiptasia core virome of 15 viral taxa from 11 viral families that was comprised of many viruses previously reported in coral viromes. Our study provides a first insight into the viral community of Aiptasia. Aiptasia seem to harbor a diverse and overall stable viral community, although certain members change in abundance when the anemone host associates with its algal endosymbiont. However, the functional significance of this remains to be determined. 


\section{Introduction}

Research in the last few decades support the notion that multicellular organisms do not live in isolation, but are forming complex relationships with a variety of microorganisms including bacteria, archaea, and viruses (McFall-Ngai et al., 2013). This entity of host organism and microorganisms is termed 'metaorganism' or 'holobiont' (Rohwer et al., 2002; Knowlton \& Rohwer, 2003; Bosch \& McFall-Ngai, 2011). Among invertebrate animal hosts, stony corals form holobionts of particular interest given they engage in endosymbioses with photosynthetic algae of the genus Symbiodinium that form the basis of coral reef ecosystems and are of high economic and ecologic importance (Muscatine \& Porter, 1977; Hoegh-Guldberg, 1999). While the cnidarian host provides a light-rich but sheltered environment, Symbiodinium supply energy-rich sugars in the form of photosynthates (Muscatine, 1967; Falkowski et al., 1984). In turn, the associated bacterial community provides functions important for nutrient cycling (Lesser \& Jarett, 2014; Rädecker et al., 2015), pathogen defense and immune system, and potentially stress resilience (Rosenberg et al., 2007; Torda et al., 2017; Ziegler et al., 2017). The importance of the viral community has become more recently the focus of research. However, the functional importance is not entirely clear, although recent studies suggest that viruses play a role in some coral diseases and potentially coral bleaching (Marhaver, Edwards \& Rohwer, 2008; Soffer et al., 2014; Weynberg et al., 2015, 2017; Correa et al., 2016; Levin et al., 2016; Brüwer et al., 2017; Vega Thurber et al., 2017).

Unfortunately, corals are under increasing threat from anthropogenic influences, in particular climate change (Hoegh-Guldberg, 1999; Hughes et al., 2003, 2017; IPCC, 2014), and understanding coral metaorganisms is critical in order to mitigate strategies to conserve coral reef ecosystems. To this end, the sea anemone Aiptasia (sensu Exaiptasia pallida) is becoming a popular model system to investigate the coral-dinoflagellate symbiosis (Weis et al., 2008; Voolstra, 2013; Baumgarten et al., 2015). While some studies looked into the association of Aiptasia with Symbiodinium (Thornhill et al., 2013; Xiang et al., 2013; Hambleton, Guse \& 
76 Pringle, 2014; Wolfowicz et al., 2016) and bacteria (Röthig et al., 2016; Herrera et al., 2017), the

77 viral community composition, to our knowledge, has not yet been investigated.

78

79 To provide a first insight into viral community composition of the cnidarian model system

80 Aiptasia, we employed a strategy used by Brüwer et al. (2017) to re-analyze a previously

81 published RNA-Seq dataset (Baumgarten et al., 2015). The transcriptomic data comprised

82 aposymbiotic Aiptasia as well as anemones partially populated and fully symbiotic with

83 endosymbiotic algae of Symbiodinium minutum (strain SSB01, Clade B1). Our strategy entailed

84 the removal of anemone host and algal endosymbiont sequences and subsequent taxonomic

85 annotation of remaining sequences to assess viral community composition and also to

86 determine whether the symbiotic state potentially influences viral association.

87 
We used a previously published RNA-Seq dataset (NCBI accessions: SRX757525 - adult, aposymbiotic Aiptasia CC7, 4 replicates; SRX757526 - adult Aiptasia CC7 partially populated with Symbiodinium minutum, 4 replicates; SRX757528 - adult Aiptasia CC7 fully symbiotic with Symbiodinium minutum, 4 replicates) of Aiptasia strain CC7 (sensu Exaiptasia pallida) generated for the purpose of assembling a reference transcriptome for the Aiptasia CC7 genome (Baumgarten et al., 2015). Animal culturing, experimental treatments, RNA extraction, and sequencing are briefly outlined below and reported in detail in Baumgarten et al. (2015).

Culturing of Aiptasia anemones and experimental treatments

Anemones of the clonal Aiptasia strain CC7 were kept in a circulating artificial seawater system at the following rearing conditions: $\sim 25^{\circ} \mathrm{C}$ with $20-40 \mu \mathrm{mol}$ photons $\mathrm{m}^{-2} \mathrm{~s}^{-1}$ photosynthetically active radiation on a $12 \mathrm{~h}: 12 \mathrm{~h}$ light:dark cycle. They were fed freshly hatched Artemia salina nauplii twice per week. In order to generate aposymbiotic anemones (i.e., without dinoflagellate symbionts), anemones were repeatedly treated with a cold-shock by transferring for $4 \mathrm{~h}$ to $4^{\circ} \mathrm{C}$ water and subsequent exposure to the photosynthesis inhibitor diuron (SigmaAldrich \#D2425) at $50 \mu \mathrm{M}$. The anemones were maintained for $\geq 1$ month in the above-detailed rearing conditions to assure no repopulation by any residual dinoflagellates. Before further treatments were applied, anemones were inspected individually via fluorescence stereomicroscopy to confirm absence of Symbiodinium. To generate, partially populated and fully symbiotic anemones, animals were kept in autoclaved and sterile-filtered artificial seawater (AFSW; other conditions as described above) and were infected with Symbiodinium minutum (strain SSB01, clade B1): day 1, algae were added at $\sim 10^{5}$ cells $/ \mathrm{ml}$; day 2, brine shrimp were added without a water change or addition of algae; day 3, AFSW was changed and algae added at $\sim 10^{5}$ cells $/ \mathrm{ml}$; day 11 , the AFSW was changed. Samples were taken at the mid-point of

114 the 12-h light period on day 0 (aposymbiotic), day 12 (partially populated), and day 30 (fully 115 symbiotic). 


\section{RNA extraction and sequencing}

118 Total RNA was extracted from the aposymbiotic, partially populated, and fully symbiotic 119 anemones (see above) using TRIzol (Life Technologies \#15596-026) following the 120 manufacturer's instructions. The mRNA was extracted from total RNA using Dynabeads 121 oligo(dT) 25 (Ambion \#61002). The quantity and quality were assessed and monitored using a 122 Bioanalyzer 2100 (Agilent Technologies, RNA Nano/Pico Chip). Subsequent library preparations 123 were conducted using the NEBNext Ultra Directional RNA Library Prep Kit (NEB \#E7420) with a 124 180-bp insert size. Libraries were sequenced together on one lane of an Illumina HiSeq2000 125 sequencer with read lengths of $2 \times 101 \mathrm{bp}$.

126

\section{Sequence data filtering}

128 The software trimmomatic (Bolger, Lohse \& Usadel, 2014) was used for quality control and read 129 trimming (settings: LEADING:30 TRAILING:30 SLIDINGWINDOW:4:30 MINLEN:35 HEADCROP:6 130 phred33). Single reads of paired-end read pairs resulting from quality control (see above) were 131 discarded and not considered for downstream analyses. Sequencing adapters were removed 132 with fastq-mcf (Aronesty, 2011) (settings: -I 35 --qual-mean 25). The BBsplit script from BBmap 133 v35 (Bushnell, 2016) was utilized to remove sequencing library spiked-in PhiX174 Illumina 134 control sequences (NCBI accession: NC_001422.1), sequences mapping to the genomes of 135 Aiptasia CC7 (NCBI accession: GCA_001417965.1) (Baumgarten et al., 2015; Liew, Aranda \& 136 Voolstra, 2016) and Symbiodinium minutum (NCBI accession: GCA_000507305.1) (Shoguchi et 137 al., 2013), as well as any sequences of 285 rRNA of sea anemones from the NCBI ' $n r^{\prime}$ database 138 (16.03.2017; search term: "(((28S) AND "cnidarians"[porgn:_txid6073]) AND "anthozoans" 139 [porgn:_txid6101]) AND "sea anemones" [porgn:_txid6103])") (settings: minid $=0.7$ local $=\mathrm{t}$ 140 qin $=33$ ). The reason for the $28 \mathrm{~S}$ rRNA removal lies in their apparent similarity to two

141 Baculoviridae, namely Choristoneura occidentalis granulovirus (ChocGV; CLARK taxonomic id:

142 364745) and Chrysodeixis chalcites nucleopolyhedrovirus (CLARK taxonomic id: 320432).

143 Retained sequence reads were used for all subsequent analyses. Overview of filters applied and 144 commands used are available as Supplementary Information (Supp. Fig. S1, Supp. Data Sheet 145 S1). 
147 Viral community analysis

148 Of the retained sequence reads (see above) only paired reads were considered and annotated 149 to the highest possible phylogenetic level using the classify_metagenome.sh script of CLARK 150 (Ounit et al., 2015) (settings: -m 0; remaining settings: default) using NCBI's RefSeq database for 151 bacteria, archaea, and viruses. The database was downloaded using the implemented 152 set_target.sh script (version 1.2.3; default settings; RefSeq release 81). Prior to normalization 153 viruses that were only annotated with one sequence in one sample (i.e., singletons) as well as 154 read pairs annotating to Choristoneura occidentalis granulovirus (ChocGV) (NCBI id: 155 NC_008168.1; CLARK taxonomic id: 364745) were removed due to similarity to 28S rRNA of sea 156 anemones (see above). For normalization, retrieved raw counts (including bacteria, archaea, 157 and viruses) were normalized using the cumulative-sum scaling (CSS) method implemented in 158 the R Bioconductor package metagenomeSeq (v 1.17.0) (Gentleman et al., 2004; Paulson et al., 159 2013; Paulson, 2014; R Core Team, 2016), and we subsequently only considered sequences that 160 were classified as of viral origin. Information on diverse groups of viruses (i.e., single strand 161 positive sense RNA ssRNA(+), single strand negative sense RNA ssRNA(-), double strand DNA 162 dsDNA, double strand RNA dsRNA, reverse transcribing RNA ssRNA(rt) as well as known virus 163 hosts (bacteria, fungi, invertebrate, vertebrate, plant, protozoan) were retrieved from either 164 the ICTV website at http://talk.ictvonline.org (Davison, 2017) or ViralZone at 165 http://viralzone.expasy.org (Hulo et al., 2011). Species richness, evenness, and Shannon-Wiener 166 Index (alpha diversity) were estimated using the R package vegan (v. $2.4-2$ ) (Oksanen et al., 167 2017). The R package ggplot2 was used for visualizing the relative abundance of viral taxa and 168 viral families (Wickham, 2016). Overview of viral community analysis and commands used are 169 available as Supplementary Information (Supp. Fig. S1, Supp. Data Sheet S1).

170 In order to test for statistical differences in the viral community composition of 171 aposymbiotic, partially populated, and fully symbiotic Aiptasia, we conducted analysis of 172 variance (ANOVA) on Pielou's evenness and Shannon-Wiener diversity. Further, we tested for 173 significant differences in relative abundance of viral taxa across conditions. To do this, we 
174 tested viral taxa $(n=116)$ with an ANOVA and a posthoc Tukey test (R Core Team, 2016) using $p$ $175<0.05$ as a cutoff.

176 To determine viromes associated with aposymbiotic, partially populated, and fully 177 symbiotic Aiptasia, we determined all viral taxa that were 100\% present across all four 178 replicates of the respective condition. Those viral taxa that were present in $100 \%$ of all 179 aposymbiotic, partially populated, and fully symbiotic Aiptasia samples were considered to be 180 core virome members. The different viromes, including the core virome, were visualized in a 181 venn diagram using BioVenn (Hulsen, de Vlieg \& Alkema, 2008). 


\section{Results}

184

\section{Viral sequence annotation}

186 A total of $297,207,704$ sequence reads (i.e., 148,603,852 paired-end read pairs) detailing four

187 replicates of adult Aiptasia anemones across each of three symbiotic stages (aposymbiotic, 188 partially populated, and fully symbiotic), i.e. total of 12 samples were available for viral 189 sequence annotation (Table 1, Supp. Fig. S1). Of those, 262,252,332 (88.24\%) sequence reads 190 were retained after quality control, read trimming, and adapter removal. After removal of 191 anemone host, algal endosymbionts, and miscellaneous other sequences (see Methods), 192 8,597,604 (2.89\%) sequence reads were available and used for bacterial, archaeal, and viral 193 annotation using the CLARK classification tool (Ounit et al., 2015). A total of 38,090 CLARK 194 classified sequences were retrieved, of which $90.97 \%$ (34,649 sequences) were of bacterial, a 195 smaller fraction of only $0.39 \%$ (148 sequences) of archaeal, and $8.65 \%(3,293$ sequences) of 196 viral origin. The virus-classified sequences comprised 116 distinct taxa covering 40 viral families 197 (Supp. Table S1).

198

\section{Aiptasia viral community composition}

200 Aiptasia was associated with a diverse viral community featuring an average species richness of 20136.72 (SD \pm 2.98 ) following Hurlbert (1971). The viral community was evenly distributed as 202 highlighted by an average Pielou's evenness of 0.90 (SD \pm 0.02 ) and Shannon-Wiener diversity 203 was 3.75 (SD \pm 0.17 ) across samples (Table 2). Measures of community composition were stable across aposymbiotic, partially populated, and fully symbiotic anemones, as neither Pielou's 205 evenness ( $p>0.88$ ) nor Shannon-Wiener diversity $(p>0.50)$ were significantly different between different symbiotic states. Almost half of the viral community was encompassed by ssRNA(+) viruses, about a third were annotated as dsDNA viruses, and less than a fifth of the community was comprised by dsRNA viruses. Conversely, ssRNA(-) and ssRNA(rt) were detected 209 at very low frequencies. The ten most abundant viral families accounted for about two-thirds of

210 the viral community (Fig. 1). The most abundant viral families included the Herpesviridae 211 (12.00\% \pm 0.49\%), Partitiviridae (9.93\% $\pm 0.30 \%)$, and Picornaviridae (9.87\% $\pm 0.45 \%)$. Generally, 
212 the community comprised few abundant and many rare viral species across treatments (Fig. 2).

213 The most abundant viral taxon, Dulcamara mottle virus $(7.16 \% \pm 0.41 \%)$, is a vertebrate virus of

214 the Tymoviridae family and belongs to the fourth most abundant viral family. The next most

215 abundant viral taxa were Caviid betaherpesvirus $2(6.48 \% \pm 0.29 \%)$, Murid betaherpesvirus 8

216 (4.34\% $\pm 0.28 \%)$, Jingmen tick virus (4.31\% $\pm 0.22 \%)$, and Bidens mottle virus (4.15\% $\pm 0.23 \%)$.

217

218 Viral communities of fully symbiotic Aiptasia are different from aposymbiotic and partially 219 populated sea anemones

220 Despite the overall similarities in viral community composition, we were interested to assess 221 whether some viral taxa were differentially abundant between symbiotic states/conditions. 222 Assessing viral taxon abundance from aposymbiotic to partially populated to fully symbiotic 223 Aiptasia revealed two general patterns (Supp. Table S1). The first pattern (hereafter referred to 224 as the 'increase'-group) included 48 viruses that increased in abundance from aposymbiotic to 225 partially populated and fully symbiotic Aiptasia (Fig. 2 A). This group was dominated by 226 Herpesviridae (7 species), Baculoviridae (5 species), and Picornaviridae (3 species). However, 227 only 13 of the 48 viral taxa assigned to this group were significantly differentially abundant, 228 including mainly viruses that are known to infect vertebrates ( 7 species) and invertebrates ( 3 229 species) (Supp. Table S2). Further, this group included a fungi-infecting species (Penicillium 230 chrysogenum virus), a plant-infecting species (Bidens mottle virus), as well as one plant- and 231 fungi-infecting viral species (White clover cryptic virus 2). The second pattern (hereafter 232 referred to as the 'decrease'-group) included 40 viral taxa that showed the opposite pattern: a 233 general decrease in relative abundance from aposymbiotic to partially populated and fully 234 symbiotic Aiptasia (Fig. 2 B) and were dominated by Picornaviridae ( 6 species), Partitiviridae (6 235 species), and Bromoviridae (3 species). However, none of the viral taxa were significantly 236 differentially abundant in this group (Supp. Table S2). The remaining viruses showed an 237 inconsistent pattern and were less frequent (28 viral taxa) (Supp. Table S2). Thus, the overall 238 viral community was rather consistent in terms of composition (Fig. 1) and abundance (Fig. 2), 239 although some viruses changed significantly in relative abundance in fully symbiotic animals, 240 but the functional significance of this remains to be determined. 
242 The Aiptasia core virome

243 Despite the overall similarities in viral community compositions (Fig. 1) and abundance (Fig. 2), 244 we were interested to assess the viromes associated with aposymbiotic, partially populated, 245 and fully symbiotic Aiptasia. To do this, we determined all viral taxa that were $100 \%$ present in 246 all four replicates of the respective condition (i.e., aposymbiotic, partially populated, and fully 247 symbiotic). Partially populated Aiptasia anemones harbored the most diverse virome consisting 248 of 41 viral species, followed by the fully symbiotic (32 viral species), and aposymbiotic virome 249 (27 viral species) (Supp. Table S3). Thus, consistent with a significant increase in relative 250 abundance for some viral taxa in fully symbiotic anemones, we also found an overall increase in 251 viral diversity. Only few viral taxa were exclusively present in one of the symbiotic states and 252 the majority of viral taxa were present in more than one symbiotic state (Fig. 3). Further, a total 253 of 15 viral taxa across 11 families comprised the Aiptasia core virome (i.e., viral taxa present in 254 100\% of all samples) (Fig. 3, Supp. Table S3). The Aiptasia core virome included the four most 255 abundant viral taxa and families, including viruses from the Herpesviridae, Partitivirdae, and 256 Picornaviridae families.

257

258 


\section{Discussion}

260

261 Despite the importance of microorganisms to their multicellular hosts (McFall-Ngai et al., 2013), 262 basic knowledge about the viral community of many organisms, including the model 263 metaorganism Aiptasia, is still lacking. The vastness of next-generation sequencing datasets 264 provides an opportunity to begin to investigate this microbial diversity, using approaches that 265 filter the target organism and classify remaining sequence reads (Brüwer et al., 2017). In this 266 study, we employed a previously generated Aiptasia RNA-Seq dataset to gain a first insight into 267 the viral community associated with Aiptasia across three different symbiotic states 268 (aposymbiotic, partially populated, fully symbiotic) with Symbiodinium. Of note, the here269 assessed RNA-Seq libraries were oligodT-selected prior to library generations. Thus, a bias 270 towards polyadenylated sequences is expected, putatively increasing our ability to detect 271 ssRNA(+) viruses that contain polyadenylated viral genomes (Adams, Antoniw \& Beaudoin, 272 2005; Le Gall et al., 2008), as well as dsDNA and ssRNA(+) viruses that polyadenylate their 273 mRNAs (Majerciak et al., 2013; te Velthuis \& Fodor, 2016). Our analysis, therefore, provides a 274 first overview of the viral community, rather than a complete characterization.

275

276

Based on our analysis, Aiptasia CC7 anemones harbor a diverse viral community that appears to 277 be similar in taxon richness compared to other non-stressed cnidarians, i.e. Hydra (Grasis et al., 278 2014). The assessed Aiptasia virome consists of 116 viral taxa from 40 viral families. 279 Interestingly, almost all of the detected viral families have been described in corals (Wood280 Charlson et al., 2015) or Symbiodinium (Brüwer et al., 2017). More specifically, 27 (in the case 281 of corals) and 32 (in the case of Symbiodinium) out of 40 detected viral families in Aiptasia in 282 this study were previously described. Firstly, this lends further support that our here-employed 283 approach works and RNA-Seq data can be queried to gain a first insight into viral diversity. 284 Secondly, it supports the notion that Aiptasia indeed is a suitable model of cnidarian285 dinoflagellate symbiosis, not only at the level of host and algal symbiont biology (Baumgarten 286 et al., 2015) but also at the level of bacteria (Röthig et al., 2016; Herrera et al., 2017) and 287 viruses (this study). 
The viral communities associated with Aiptasia are dominated by Herpesviridae (vertebrate-infecting), Partitiviridae (plant- and fungi -infecting), and Picornaviridae 290 (vertebrate-infecting) (Hulo et al., 2011) (Fig. 1), which is of particular notice, given that Aiptasia 291 is an invertebrate. However, vertebrate viruses have been frequently found in cnidarian 292 viromes (Grasis et al., 2014; Wood-Charlson et al., 2015; Vega Thurber et al., 2017). A case 293 study on the freshwater polyp Hydra, Grasis et al. (2014) suggested that the increased 294 vertebrate-virus abundance might be due to a variety of ancestral genes that have been lost in 295 other invertebrates, such as Drosophila melanogaster and Caenorhabditis elegans), as well as a 296 great similarity of the genome organization. Despite these evolutionary considerations, caution 297 has to be applied when categorizing viruses as vertebrate-, invertebrate-, or fungi-infection, etc. 298 as this categorization is mainly based on previous findings and descriptions and might not have 299 a claim to completeness. Last, the uneven presentation of viruses from different host organisms 300 in viral databases might further contribute to uncertainties regarding these categorizations.

301 Despite our finding of an overall diverse and stable viral community associated with 302 Aiptasia, we were interested to further assess whether the viral community is different under 303 different symbiotic states (i.e., aposymbiotic, partially populated, and fully symbiotic). This 304 would further contribute to our understanding of the intricacies of the cnidarian-dinoflagellate 305 symbiosis (Mies et al., 2017) and provide putative important detail concerning the role of 306 viruses in this symbiosis. We find that viral diversity and community composition remains 307 overall stable, irrespective of the symbiotic state with Symbiodinium minutum. However, 308 individual viral taxa change in abundance across symbiotic states. Most noticeably, we find 309 significant abundance increases of 13 viral taxa when the host animal becomes partially 310 populated and fully infected with Symbiodinium (Fig. 2 A). We initially hypothesized that 311 members of the 'increase'-group would be dominated by plant-infecting viruses, given that 312 Symbiodinium may come associated with its distinct set of viruses. However, we mainly 313 observed vertebrate-infecting viruses, mostly Herpesviridae, as well as some invertebrate 314 viruses to increase in abundance. In contrast to the 'increase'-group, the 'decrease'-group (Fig. $3152 \mathrm{~B}$ ) is comprised of viruses that decrease in abundance and is dominated by plant- and fungi316 infecting viral species of the Partitiviridae family, as well as other plant-infecting viruses, such as 
317 members of the Virgaviridae and Bromoviridae families, and vertebrate viruses, mainly of the

318 Picornaiviridae family. Notably, none of these changes were significant. As such, it remains to

319 be determined whether some viruses increase, whereas other viruses decrease upon entering 320 partially populated or fully symbiotic states. However, it is tempting to speculate that the viral 321 community might compensate and adapt to the changing environment, as suggested earlier for 322 the Hydra virome (Grasis et al., 2014).

323

324 To better understand the contribution of the virome to a metaorganism, knowledge about the 325 constantly associated viruses (i.e., viral taxa of the core virome) might provide further clues to 326 their importance and ecological significance. A case study in Hydra assessed the viral 327 community composition of four different Hydra strains and concluded that the virome, similar 328 to the microbiome, is species-specific (Grasis et al., 2014). Presuming a similar pattern for 329 Aiptasia, we aimed to identify permanent members of the viral community. In our study, 330 viruses that were present in all samples were considered members of the core virome and, 331 thus, suggested to be permanent members of the viral community. Interestingly, plant- and 332 vertebrate-infecting viruses dominate the here-identified Aiptasia core virome. The Aiptasia core virome comprises 15 viral species from 11 viral families, which is in 334 line with a recent review by Vega Thurber et al. (2017) proposing between 9 and 12 viral 335 families as members of a coral core virome. More specifically, viruses of the Mimivirdae, 336 Herpesviridae, and Poxviridae families were suggested to be part of the coral core virome (Vega 337 Thurber et al., 2017) and are also present in the Aiptasia core virome. In addition, viruses 338 similar to the Herpesviridae family have been described in almost all studies investigating the 339 viral community of anthozoans (Grasis et al., 2014; Wood-Charlson et al., 2015; Vega Thurber et 340 al., 2017) including this study, and thus, are most likely important members of the cnidarians 341 metaorganism. Bacteriophages of the order Caudovirales (including Siphoviridae, Podoviridae, 342 and Myoviridae), which are most abundant members of the Hydra virome (Grasis et al., 2014) 343 and are frequently present in coral viromes (Wood-Charlson et al., 2015), were, however, 344 absent in the Aiptasia core virome. Taken together, despite some differences to other 345 anthozoan viromes, which may be partially attributed to a bias stemming from our approach to 
346 use RNA-Seq data, the Aiptasia viral community exhibits a comparable complexity and harbors

347 a large similarity in composition compared to anthozoan core viromes. Henceforth, our 348 analyses support Aiptasia as a model metaorganism to study not only the cnidarian349 dinoflagellate symbiosis but also the role of associated viruses with potential implications for 350 coral health.

351

352 Conclusions

353 Although the power and validity of the metaorganism concept receive growing attention, we 354 know little about the viral communities associated with many animals and host, in particular of 355 corals and other marine invertebrates. To further complement the usability and resources 356 available for the Aiptasia model system, we annotated RNA-Seq data to describe the virome 357 associated with aposymbiotic, partially populated, and fully symbiotic Aiptasia. We find that 358 Aiptasia is associated with a diverse and stable viral community. Certain viral taxa of this 359 community increase their abundance when aposymbiotic anemones establish a symbiotic 360 relationship with their endosymbiont Symbiodinium. Hence, the viral community responds to 361 the symbiosis suggesting putative functional implications that need to be assessed in future 362 studies. Further, we identified candidate members of the Aiptasia core virome comprised of 363 viruses from the families Mimiviridae, Heperesviridae, and Poxviridae families that resembles 364 the composition of coral core viromes. The Aiptasia model metaorganism may facilitate 365 targeted studies to investigate the ecological importance of viruses the cnidarian-dinoflagellate 366 endosymbiosis with implications for coral reef health. 


\section{Acknowledgements}

369 JDB was funded by a Visiting Student Research Program (VSRP) fellowship awarded by King 370 Abdullah University of Science and Technology (KAUST). Additional supported was provided by 371 baseline funds from KAUST to CRV. The funders had no role in the design of the study and 372 collection, analysis, and interpretation of data, and in writing the manuscript.

373

\section{Author contribution}

375 JDB and CRV designed and conceived the study. JDB generated data. JDB and CRV analyzed 376 data. JDB and CRV wrote the manuscript.

377

\section{List of abbreviations}

379 AFSW sterile-filtered artificial sea-water

380 bp base pairs

381 ChocGV Choristoneura occidentalis granulovirus

382 dsDNA double-stranded DNA virus

383 dsRNA double-stranded RNA virus

384 RNA-Seq RNA-sequencing

385 rRNA ribosomal RNA

386 ssRNA(+) positive-sense single-stranded RNA virus

387 ssRNA(-) negative-sense single-stranded RNA virus

388 ssRNA(rt) reverse-transcribing single-stranded RNA virus 


\section{References}

Adams MJ., Antoniw JF., Beaudoin F. 2005. Overview and analysis of the polyprotein cleavage sites in the family Potyviridae. Molecular Plant Pathology 6:471-487. DOI: 10.1111/j.13643703.2005.00296.x.

Aronesty E. 2011. ea-utils: Command-line tools for processing biological sequencing data. Expression Analysis, Durham, NC.

Baumgarten S., Simakov O., Esherick LY., Liew YJ., Lehnert EM., Michell CT., Li Y., Hambleton EA., Guse A., Oates ME., Gough J., Weis VM., Aranda M., Pringle JR., Voolstra CR. 2015. The genome of Aiptasia, a sea anemone model for coral symbiosis. Proceedings of the National Academy of Sciences of the United States of America 112:11893-11898. DOI: 10.1073/pnas.1513318112.

Bolger AM., Lohse M., Usadel B. 2014. Trimmomatic: A flexible trimmer for Illumina sequence data. Bioinformatics 30:2114-2120. DOI: 10.1093/bioinformatics/btu170.

Bosch TCG., McFall-Ngai MJ. 2011. Metaorganisms as the new frontier. Zoology 114:185-190. DOI: 10.1016/j.zool.2011.04.001.

Brüwer JD., Agrawal S., Liew YJ., Aranda M., Voolstra CR. 2017. Association of coral algal symbionts with a diverse viral community responsive to heat shock. BMC Microbiology 17:174. DOI: 10.1186/s12866-017-1084-5.

Bushnell B. 2016. BBMap short read aligner. University of California, Berkeley, California. URL http://sourceforge. net/projects/bbmap.

Correa AMS., Ainsworth TD., Rosales SM., Thurber AR., Butler CR., Vega Thurber RL. 2016. Viral outbreak in corals associated with an in situ bleaching event: Atypical herpes-like viruses and a new megavirus infecting symbiodinium. Frontiers in Microbiology 7:1-14. DOI: 10.3389/fmicb.2016.00127.

Davison AJ. 2017. Journal of general virology - Introduction to "ICTV virus taxonomy profiles." Journal of General Virology 98:1. DOI: 10.1099/jgv.0.000686.

Falkowski PG., Dubinsky Z., Muscatine L., Porter JW. 1984. Light and the Bioenergetics of a Symbiotic Coral. Source: BioScience 34:705-709.

Le Gall O., Christian P., Fauquet CM., King AMQ., Knowles NJ., Nakashima N., Stanway G., 
Gorbalenya AE. 2008. Picornavirales, a proposed order of positive-sense single-stranded RNA viruses with a pseudo-T = 3 virion architecture. Archives of Virology 153:715-727. DOI: $10.1007 / \mathrm{s} 00705-008-0041-x$.

422 Gentleman R., Carey V., Bates D., Bolstad B., Dettling M., Dudoit S., Ellis B., Gautier L., Ge Y., 423 Gentry J., Hornik K., Hothorn T., Huber W., lacus S., Irizarry R., Leisch F., Li C., Maechler M., 424 Rossini A., Sawitzki G., Smith C., Smyth G., Tierney L., Yang J., Zhang J. 2004. Bioconductor: 425 open software development for computational biology and bioinformatics. Genome 426 Biology 5:R80. DOI: 10.1186/gb-2004-5-10-r80.

427 Grasis JA., Lachnit T., Anton-Erxleben F., Lim YW., Schmieder R., Fraune S., Franzenburg S., Insua 428 S., Machado G., Haynes M., Little M., Kimble R., Rosenstiel P., Rohwer FL., Bosch TCG. 2014. Species-specific viromes in the ancestral holobiont hydra. PLOS ONE 9:e109952. DOI: 10.1371/journal.pone.0109952.

431

432 433

434

435

436

437

438

439

440

441

442

443

444

445

446

447

Hambleton EA., Guse A., Pringle JR. 2014. Similar specificities of symbiont uptake by adults and larvae in an anemone model system for coral biology. The Journal of Experimental Biology 217:1613-1619. DOI: 10.1242/jeb.095679.

Herrera M., Ziegler M., Voolstra CR., Aranda Lastra MI. 2017. Laboratory-cultured strains of the sea anemone Exaiptasia reveal distinct bacterial communities. Frontiers in Marine Science 4:115.

Hoegh-Guldberg O. 1999. Climate Change, coral bleaching and the future of the world's coral reef. CSIRO Australia 50:839-66. DOI: 10.1071/MF00030.

Hughes TP., Baird AH., Bellwood DR., Card M., Connolly SR., Folke C., Grosberg R., HoeghGuldberg O., Jackson JBC., Kleypas J. 2003. Climate change, human impacts, and the resilience of coral reefs. Science 301:929-933. DOI: 10.1126/science.1085046.

Hughes TP., Kerry JT., Álvarez-Noriega M., Álvarez-Romero JG., Anderson KD., Baird AH., Babcock RC., Beger M., Bellwood DR., Berkelmans R. 2017. Global warming and recurrent mass bleaching of corals. Nature 543:373-377. DOI: 10.1038/nature21707.

Hulo C., De Castro E., Masson P., Bougueleret L., Bairoch A., Xenarios I., Le Mercier P. 2011. ViralZone: a knowledge resource to understand virus diversity. Nucleic Acids Research 39:D576-D582. 
448 Hulsen T., de Vlieg J., Alkema W. 2008. BioVenn - a web application for the comparison and 449 visualization of biological lists using area-proportional Venn diagrams. BMC Genomics 450 9:488. DOI: 10.1186/1471-2164-9-488.

451 Hurlbert SH. 1971. The nonconcept of species diversity: a critique and alternative parameters. $452 \quad$ Ecology 52:577-586. DOI: 10.2307/1934145.

453 IPCC. 2014. Climate change 2014: synthesis report. Contribution of Working Groups I, II and III to 454 the fifth assessment report of the Intergovernmental Panel on Climate Change.

455 Knowlton N., Rohwer F. 2003. Multispecies microbial mutualisms on coral reefs: the host as a 456 habitat. the american naturalist 162:S51-S62. DOI: doi.org/10.1086/378684.

457 Lesser MP., Jarett JK. 2014. Culture-dependent and culture-independent analyses reveal no 458 prokaryotic community shifts or recovery of Serratia marcescens in Acropora palmata with 459 white pox disease. FEMS Microbiology Ecology 88:457-467. DOI: 10.1111/1574$460 \quad 6941.12311$.

461 Levin RA., Voolstra CR., Weynberg KD., van Oppen MJH. 2016. Evidence for a role of viruses in 462 the thermal sensitivity of coral photosymbionts. The ISME Journal 11:808-812. DOI: $463 \quad$ 10.1038/ismej.2016.154.

464 Liew YJ., Aranda M., Voolstra CR. 2016. Reefgenomics. Org-a repository for marine genomics 465 data. Database 2016:baw152.

466 Majerciak V., Ni T., Yang W., Meng B., Zhu J., Zheng ZM. 2013. A Viral Genome Landscape of 467 RNA Polyadenylation from KSHV Latent to Lytic Infection. PLoS Pathogens 9:e1003749. $468 \quad$ DOI: 10.1371/journal.ppat.1003749.

469 Marhaver KL., Edwards RA., Rohwer F. 2008. Viral communities associated with healthy and $470 \quad$ bleaching corals. Environmental Microbiology 10:2277-2286. DOI: 10.1111/j.1462$471 \quad 2920.2008 .01652 . x$.

472 McFall-Ngai M., Hadfield MG., Bosch TCG., Carey H V., Domazet-Lošo T., Douglas AE., Dubilier 473 N., Eberl G., Fukami T., Gilbert SF., Hentschel U., King N., Kjelleberg S., Knoll AH., Kremer 474 N., Mazmanian SK., Metcalf JL., Nealson K., Pierce NE., Rawls JF., Reid A., Ruby EG., 475 Rumpho M., Sanders JG., Tautz D., Wernegreen JJ. 2013. Animals in a bacterial world, a 476 new imperative for the life sciences. Proceedings of the National Academy of Sciences 
110:3229-3236. DOI: 10.1073/pnas.1218525110.

478 Mies M., Sumida PYG., Rädecker N., Voolstra CR. 2017. Marine Invertebrate Larvae Associated with Symbiodinium: A Mutualism from the Start? Frontiers in Ecology and Evolution 5:111. DOI: $10.3389 /$ fevo.2017.00056.

481

482

483

484

485

486

487

488

489

490

491

492

493

494

495

496

497

498

499

500

501

502

503

504

505

Muscatine L. 1967. Glycerol Excretion by symbiotic algae from corals and tridacna and its control by the host. Science 156:516-519. DOI: 10.1126/science.156.3774.516.

Muscatine L., Porter JW. 1977. Reef corals: mutualistic symbioses adapted to nutrient-poor environments. Bioscience 27:454-460. DOI: doi.org/10.2307/1297526.

Oksanen J., Blanchet FG., Friendly M., Kindt R., Legendre P., McGlinn D., Minchin PR., O’Hara RB., Simpson GL., Solymos P., Henry M., Stevens H., Szoecs E., Wagner H. 2017. Vegan: Community Ecology Package. 2017. R-package version 2.4-2.

Ounit R., Wanamaker S., Close TJ., Lonardi S. 2015. CLARK: fast and accurate classification of metagenomic and genomic sequences using discriminative k-mers. BMC Genomics 16:236. DOI: 10.1186/s12864-015-1419-2.

Paulson J. 2014. metagenomeSeq: Statistical analysis for sparse high-throughput sequencing. Bioconductor.Jp:1-20.

Paulson JN., Stine OC., Bravo HC., Pop M. 2013. Differential abundance analysis for microbial marker-gene surveys. Nature Methods 10:1200-2. DOI: 10.1038/nmeth.2658.

R Core Team. 2016. R: A language and environment for statistical computing. Vienna: R Foundation for Statistical Computing, Vienna, Austria.

Rädecker N., Pogoreutz C., Voolstra CR., Wiedenmann J., Wild C. 2015. Nitrogen cycling in corals: The key to understanding holobiont functioning? Trends in Microbiology 23:490497. DOI: 10.1016/j.tim.2015.03.008.

Rohwer F., Seguritan V., Azam F., Knowlton N. 2002. Diversity and distribution of coralassociated bacteria. Marine Ecology Progress Series 243:1-10. DOI: 10.3354/meps243001.

Rosenberg E., Koren O., Reshef L., Efrony R., Zilber-Rosenberg I. 2007. The role of microorganisms in coral health, disease and evolution. Nature Reviews Microbiology 5:355-362. DOI: 10.1038/nrmicro1635.

Röthig T., Costa RM., Simona F., Baumgarten S., Torres AF., Radhakrishnan A., Aranda M., 
506

507

508

509

510

511

512

513

514

515

516

517

518

519

520

521

522

523

524

525

526

527

528

529

530

531

532

533

534

Voolstra CR. 2016. Distinct Bacterial Communities Associated with the Coral Model Aiptasia in Aposymbiotic and Symbiotic States with Symbiodinium. Frontiers in Marine Science 3:234. DOI: 10.3389/fmars.2016.00234.

Shoguchi E., Shinzato C., Kawashima T., Gyoja F., Mungpakdee S., Koyanagi R., Takeuchi T., Hisata K., Tanaka M., Fujiwara M. 2013. Draft assembly of the Symbiodinium minutum nuclear genome reveals dinoflagellate gene structure. Current biology 23:1399-1408.

Soffer N., Brandt ME., Correa AMS., Smith TB., Thurber RV. 2014. Potential role of viruses in white plague coral disease. The ISME Journal 8:271-283. DOI: 10.1038/ismej.2013.137.

Thornhill DJ., Xiang Y., Pettay DT., Zhong M., Santos SR. 2013. Population genetic data of a model symbiotic cnidarian system reveal remarkable symbiotic specificity and vectored introductions across ocean basins. Molecular Ecology 22:4499-4515. DOI: 10.1111/mec.12416.

Torda G., Donelson JM., Aranda M., Barshis DJ., Bay L., Berumen ML., Bourne DG., Cantin N., Foret S., Matz M. 2017. Rapid adaptive responses to climate change in corals. Nature Climate Change 7:627-636. DOI: 10.1038/NCLIMATE3374.

Vega Thurber R., Payet JP., Thurber AR., Correa AMS. 2017. Virus-host interactions and their roles in coral reef health and disease. Nature Reviews Microbiology 15:205-216. DOI: 10.1038/nrmicro.2016.176.

te Velthuis AJW., Fodor E. 2016. Influenza virus RNA polymerase: insights into the mechanisms of viral RNA synthesis. Nature Reviews Microbiology 14:479-493. DOI: 10.1038/nrmicro.2016.87.

Voolstra CR. 2013. A journey into the wild of the cnidarian model system Aiptasia and its symbionts. Molecular Ecology 22:4366-4368. DOI: 10.1111/mec.12464.

Weis VM., Davy SK., Hoegh-Guldberg O., Rodriguez-Lanetty M., Pringle JR. 2008. Cell biology in model systems as the key to understanding corals. Trends in Ecology and Evolution 23:369-376. DOI: doi.org/10.1016/j.tree.2008.03.004.

Weynberg KD., Levin RA., Neave MJ., Clode PL., Voolstra CR., Brownlee C., Laffy PW., Webster NS., Wood-charlson EM., Oppen JH Van., Biology PF., Cluster CC., Sea R., Science E., Arabia S., Resources B. 2017. Prevalent viral infection in cultures of the coral algal endosymbiont 

Symbiodinium. Coral Reefs 36:773-784. DOI: 10.1007/s00338-017-1568-7.

536 Weynberg KD., Voolstra CR., Neave MJ., Buerger P., van Oppen MJH. 2015. From cholera to 537 corals: Viruses as drivers of virulence in a major coral bacterial pathogen. Scientific Reports $538 \quad$ 5:17889. DOI: 10.1038/srep17889.

539 Wickham H. 2016. ggplot2: elegant graphics for data analysis. Cham: Springer.

540 Wolfowicz I., Baumgarten S., Voss PA., Hambleton EA., Voolstra CR., Hatta M., Guse A. 2016.

541 Aiptasia sp. larvae as a model to reveal mechanisms of symbiont selection in cnidarians.

542 Scientific Reports 6:32366. DOI: 10.1038/srep32366.

543 Wood-Charlson EM., Weynberg KD., Suttle C a., Roux S., van Oppen MJH. 2015. Metagenomic 544 characterisation of viral communities in corals: Mining biological signal from

545 methodological noise. Environmental Microbiology 17:1-21. DOI: 10.1111/1462-

5462920.12803.

547 Xiang T., Hambleton EA., Denofrio JC., Pringle JR., Grossman AR. 2013. Isolation of clonal axenic 548 strains of the symbiotic dinoflagellate Symbiodinium and their growth and host $549 \quad$ specificity1. Journal of Phycology 49:447-458. DOI: 10.1111/jpy.12055.

550 Ziegler M., Seneca FO., Yum LK., Palumbi SR., Voolstra CR. 2017. Bacterial community dynamics 551 are linked to patterns of coral heat tolerance. Nature Communications 8:14213. DOI:

$552 \quad 10.1038 /$ ncomms14213.

553 


\section{Tables}

555

556 Table 1. Sequence data overview and read-based annotation. Numbers of raw and retained 557 (i.e., after quality filtering, trimming, and removal of host anemones, symbiont algae, PhiX, 28S 558 rRNA) sequence reads, as well as number of annotated read pairs are provided. Retained 559 sequence reads were used for taxonomic analysis. Apo = aposymbiotic; Partial = partially 560 populated (after 12 days of infection); Symbiotic = fully symbiotic (fully infected, after 30 days 561 of infection). R1 - R4 = replicated anemones.

562

563 Table 2. Overview of Aiptasia viral community richness, evenness, diversity, and most

564 abundant viral taxon. Species richness was estimated following Hurlbert (1971) after rarefying 565 to the lowest number of viral-annotated sequences $(n=82)$. Apo $=$ aposymbiotic; Partial $=$ 566 partially populated (after 12 days of infection); Symbiotic = fully symbiotic (fully infected, after 56730 days of infection). R1 - R4 = replicated anemones. 
Figures

570

571 Figure 1. Aiptasia viral community composition. Shown are the 10 most abundant viral families 572 associated with adult Aiptasia anemones across three symbiotic stages (aposymbiotic, partially 573 populated, and fully symbiotic); remaining viruses are associated under 'Others'. Apo = 574 aposymbiotic; Partially = partially populated (after 12 days of infection); Sym = fully symbiotic

575 (fully infected, after 30 days of infection). 1 - 4 = replicated anemones.

576

577 Figure 2. Relative abundance changes of viruses associated with Aiptasia in relation to 578 aposymbiotic, partially populated, and fully symbiotic anemones. Viral taxa could be 579 separated into two groups: (A) viral taxa that increased in abundance from aposymbiotic to 580 partially populated and fully symbiotic Aiptasia ('increase' group); (B) viral taxa that showed a 581 general decrease in abundance from aposymbiotic to partially populated and fully symbiotic 582 Aiptasia.

583

584 Figure 3. Viromes associated with aposymbiotic, partially populated, and fully symbiotic 585 Aiptasia. All viral taxa present in $100 \%$ across all four replicates of the respective state (i.e., 586 aposymbiotic (red area), partially populated (yellow area), and fully symbiotic (blue area)) were 587 considered virome members. The core virome (dark gray area) denotes the intersection of 588 viromes from aposymbiotic, partially populated, and fully symbiotic anemones: 15 viral taxa 589 were present in $100 \%$ of all samples and are proposed members of the Aiptasia core virome. 590 The areas correspond proportionally to the number of viral taxa they encompass. 
591

592

593 Supp. Table S1. CSS normalized sequence counts for all annotated viruses. The viral 594 spreadsheet is completed with genome organization information and information about 595 respective hosts. A1M - A4M: aposymbiotic; I1M - I4M = partially populated (after 12 days of 596 infection); S1M - S4M = fully symbiotic (fully infected, after 30 days of infection).

597

598 Supp. Table S2. Abundance changes of viruses associated with Aiptasia in relation to 599 aposymbiotic, partially populated, and fully symbiotic anemones. Shown are viral taxa tested, 600 ANOVA p-values (significant values in bold), and associated post-hoc Tukey tests, as well as 601 assortment to the 'increase' and 'decrease' group.

602

603

604

605

606

607

608

609

610 Supp. Figure S1. Overview of bioinformatics pipeline.

611

\section{Supplementary Information}

Supp. Table S3. Aiptasia core virome and viromes associated with aposymbiotic, partially populated, and fully symbiotic anemones of Aiptasia. Only viruses present in $100 \%$ of each respective symbiotic state were considered. Viruses present across all samples comprise the core virome. Members of the core virome are highlighted in bold.

Supp. Data Sheet S1. List of bioinformatics software and commands used. 
Table $\mathbf{1}$ (on next page)

Table 1. Sequence data overview and read-based annotation. 
1 Table 1. Sequence data overview and read-based annotation. Numbers of raw and retained 2 (i.e., after quality filtering, trimming, and removal of host anemones, symbiont algae, PhiX, 28S

3 rRNA) sequence reads, as well as number of annotated read pairs are provided. Retained 4 sequence reads were used for taxonomic analysis. Apo = aposymbiotic; Partial $=$ partially 5 populated (after 12 days of infection); Symbiotic = fully symbiotic (fully infected, after 30 days 6 of infection). R1 - R4 = replicated anemones.

7

\begin{tabular}{llrrrrrr} 
Condition & Sample & Raw reads & \multicolumn{1}{c}{$\begin{array}{c}\text { Retained } \\
\text { reads }\end{array}$} & $\begin{array}{c}\text { Classified } \\
\text { read pairs } \\
\text { (total) }\end{array}$ & $\begin{array}{c}\text { Classified } \\
\text { read pairs } \\
\text { (virus) }\end{array}$ & $\begin{array}{c}\text { Classified } \\
\text { read pairs } \\
\text { (bacteria) }\end{array}$ & $\begin{array}{r}\text { Classified } \\
\text { read pairs } \\
\text { (archaea) }\end{array}$ \\
\hline Apo & R1 & $23,314,626$ & 633,310 & 2,220 & 82 & 2,136 & 2 \\
& R2 & $21,623,164$ & 640,332 & 2,176 & 203 & 1,965 & 8 \\
& R3 & $23,905,820$ & 702,856 & 3,413 & 199 & 3,206 & 8 \\
& R4 & $23,200,990$ & 803,114 & 8,407 & 552 & 7,840 & 15 \\
Partial & R1 & $21,485,094$ & 798,846 & 8,752 & 733 & 7,980 & 39 \\
& R2 & $23,355,938$ & 657,924 & 2,215 & 232 & 1,973 & 10 \\
& R3 & $26,458,678$ & 665,100 & 2,318 & 207 & 2,099 & 12 \\
& R4 & $33,532,640$ & 818,942 & 2,743 & 277 & 2,452 & 14 \\
Symbiotic & R1 & $23,292,594$ & 653,802 & 1,172 & 171 & 996 & 5 \\
& R2 & $25,013,812$ & 684,102 & 1,516 & 220 & 1,286 & 10 \\
& R3 & $24,218,018$ & 704,760 & 1,284 & 165 & 1,112 & 7 \\
& R4 & $27,806,330$ & 834,516 & 1,874 & 252 & 1,604 & 18 \\
Total & & $297,207,704$ & $8,597,604$ & 38,090 & 3,293 & 34,649 & 148 \\
Percentage & & & & & $8.65 \%$ & $90.97 \%$ & $0.39 \%$
\end{tabular}


Table 2 (on next page)

Table 2. Overview of Aiptasia viral community richness, evenness, diversity, and most abundant viral taxon. 
1 Table 2. Overview of Aiptasia viral community richness, evenness, diversity, and most

2 abundant viral taxon. Species richness was estimated following Hurlbert (1971) after rarefying

3 to the lowest number of viral-annotated sequences $(n=82)$. Apo $=$ aposymbiotic; Partial $=$

4 partially populated (after 12 days of infection); Symbiotic = fully symbiotic (fully infected, after

530 days of infection). R1 - R4 = replicated anemones.

6

\begin{tabular}{llcccr} 
Condition & Replicate & $\begin{array}{c}\text { Species richness } \\
\text { (Hurlbert) }\end{array}$ & $\begin{array}{c}\text { Evenness } \\
\text { (Pielou) }\end{array}$ & $\begin{array}{c}\text { Shannon-Wiener } \\
\text { Diversity Index }\end{array}$ & $\begin{array}{c}\text { Most abundant } \\
\text { viral taxon }\end{array}$ \\
\hline Apo & R1 & 29.264 & 0.911 & 3.312 & $15.85 \%$ \\
& R2 & 36.623 & 0.908 & 3.749 & $8.37 \%$ \\
& R3 & 37.751 & 0.914 & 3.803 & $8.04 \%$ \\
& R4 & 36.821 & 0.863 & 3.792 & $7.07 \%$ \\
Partial & R1 & 35.387 & 0.853 & 3.727 & $9.41 \%$ \\
& R2 & 35.039 & 0.890 & 3.660 & $10.78 \%$ \\
& R3 & 39.679 & 0.921 & 3.900 & $7.73 \%$ \\
& R4 & 39.386 & 0.901 & 3.902 & $7.22 \%$ \\
& R1 & 34.496 & 0.877 & 3.606 & $10.53 \%$ \\
& R2 & 37.559 & 0.903 & 3.784 & $10.00 \%$ \\
& R3 & 38.547 & 0.905 & 3.820 & $10.91 \%$ \\
& R4 & 40.108 & 0.904 & 3.915 & $8.73 \%$
\end{tabular}




\section{Figure 1 (on next page)}

Figure 1. Aiptasia viral community composition.

Shown are the 10 most abundant viral families associated with adult Aiptasia anemones across three symbiotic stages (aposymbiotic, partially populated, and fully symbiotic); remaining viruses are associated under 'Others'. Apo = aposymbiotic; Partially = partially populated (after 12 days of infection); Sym = fully symbiotic (fully infected, after 30 days of infection). 1 - 4 = replicated anemones. 
Aposymbiotic
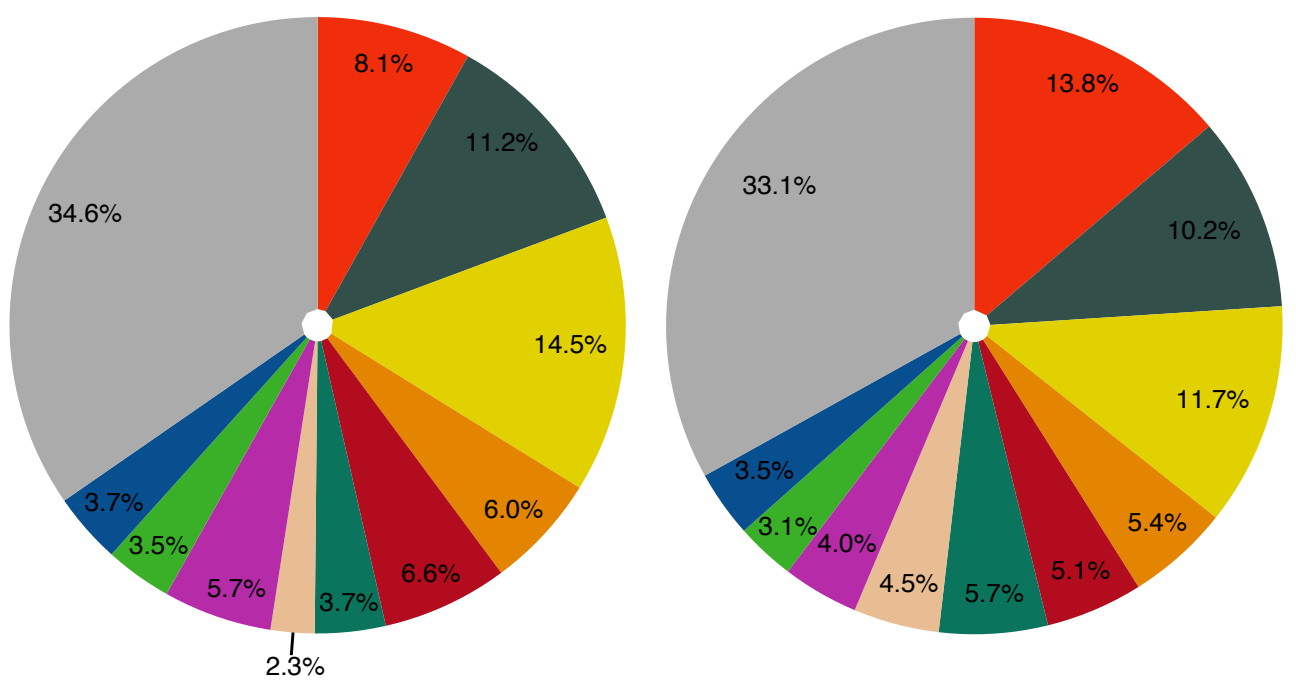

Symbiotic

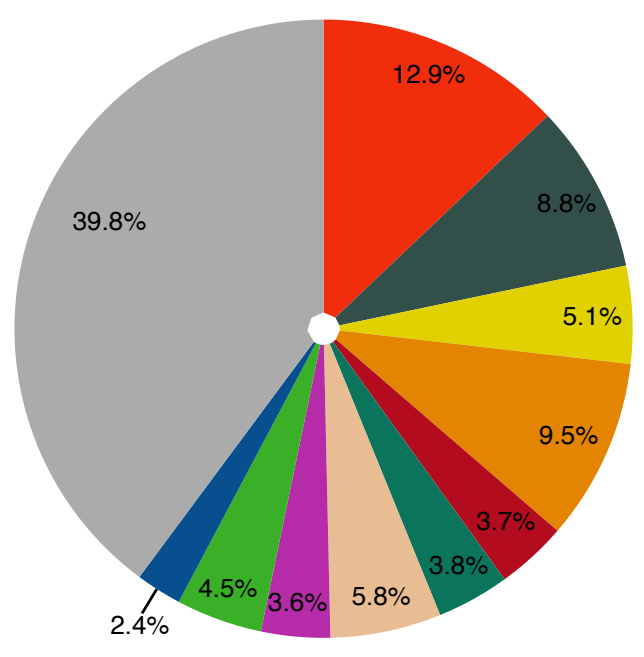

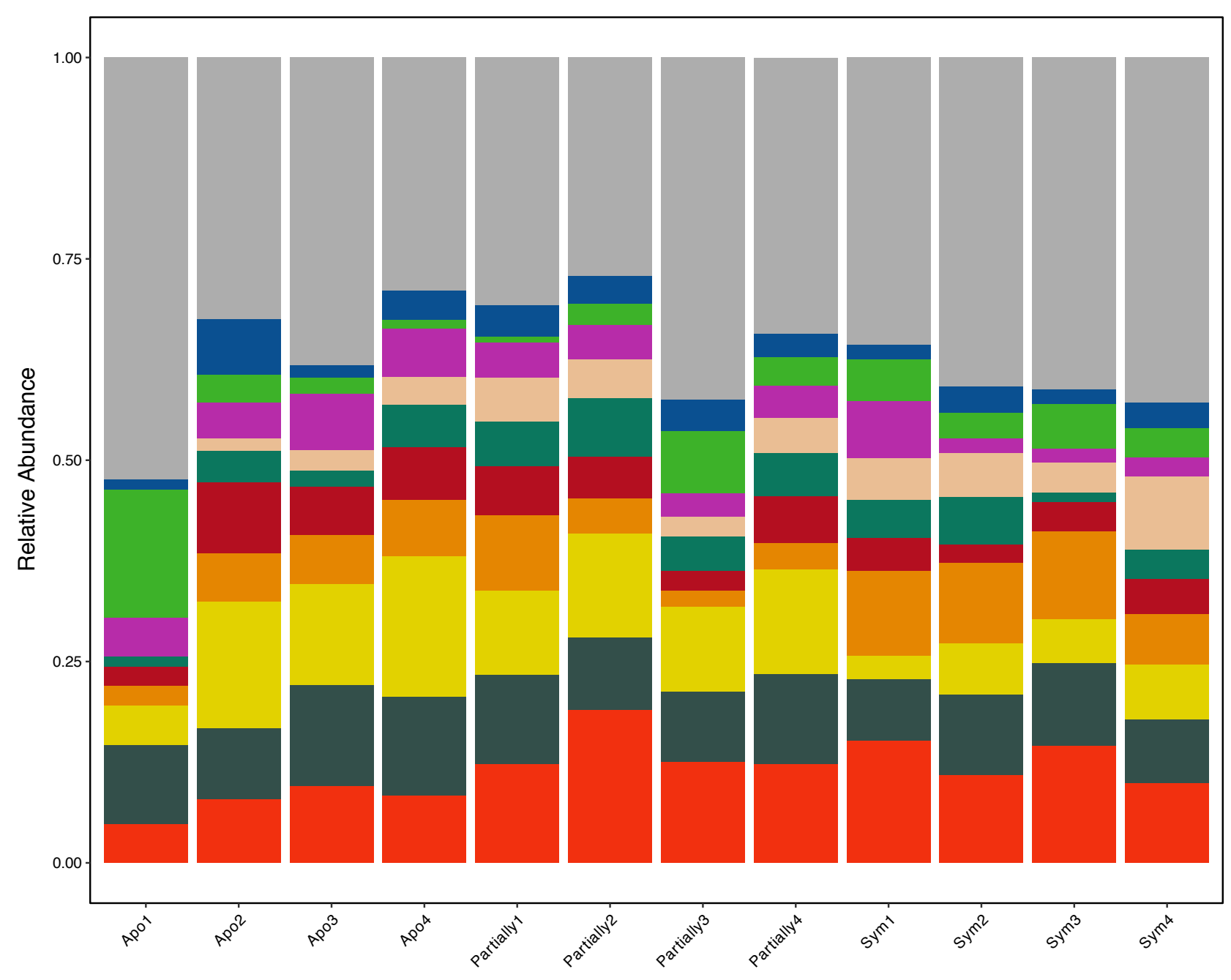

\begin{tabular}{|l|}
\hline \multicolumn{1}{|c|}{ Family } \\
Others \\
Astroviridae \\
\hline Mimiviridae \\
Polydnaviridae \\
Potyviridae \\
Hepeviridae \\
Bromoviridae \\
Tymoviridae \\
Picornaviridae \\
Partitiviridae \\
Herpesviridae \\
\hline
\end{tabular}




\section{Figure 2 (on next page)}

Figure 2. Relative abundance changes of viruses associated with Aiptasia in relation to aposymbiotic, partially populated, and fully symbiotic anemones.

Viral taxa could be separated into two groups: (A) viral taxa that increased in abundance from aposymbiotic to partially populated and fully symbiotic Aiptasia ('increase' group); (B) viral taxa that showed a general decrease in abundance from aposymbiotic to partially populated and fully symbiotic Aiptasia. 




Ш

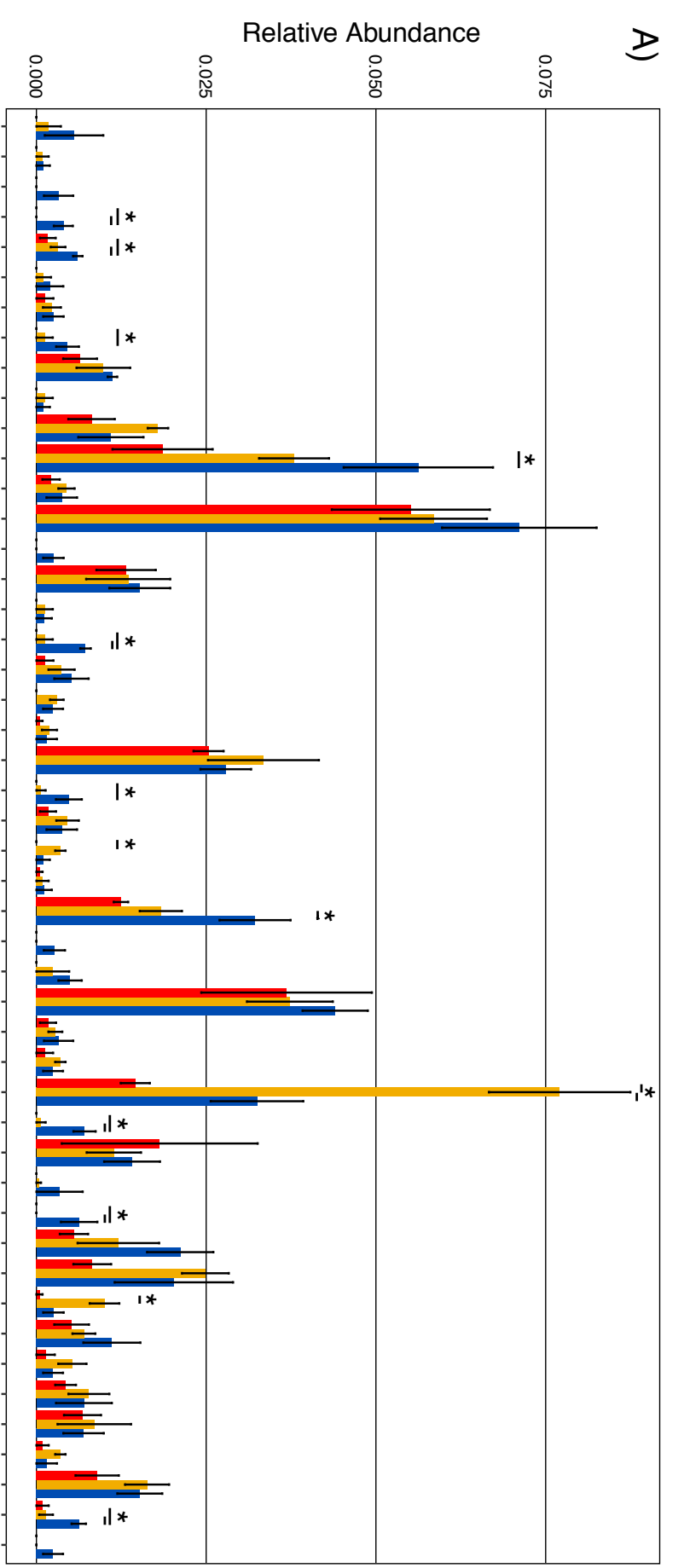




\section{Figure 3 (on next page)}

Figure 3. Viromes associated with aposymbiotic, partially populated, and fully symbiotic Aiptasia.

All viral taxa present in $100 \%$ across all four replicates of the respective state (i.e., aposymbiotic (red area), partially populated (yellow area), and fully symbiotic (blue area)) were considered virome members. The core virome (dark gray area) denotes the intersection of viromes from aposymbiotic, partially populated, and fully symbiotic anemones: 15 viral taxa were present in $100 \%$ of all samples and are proposed members of the Aiptasia core virome. The areas correspond proportionally to the number of viral taxa they encompass. 


\section{Partially Populated}

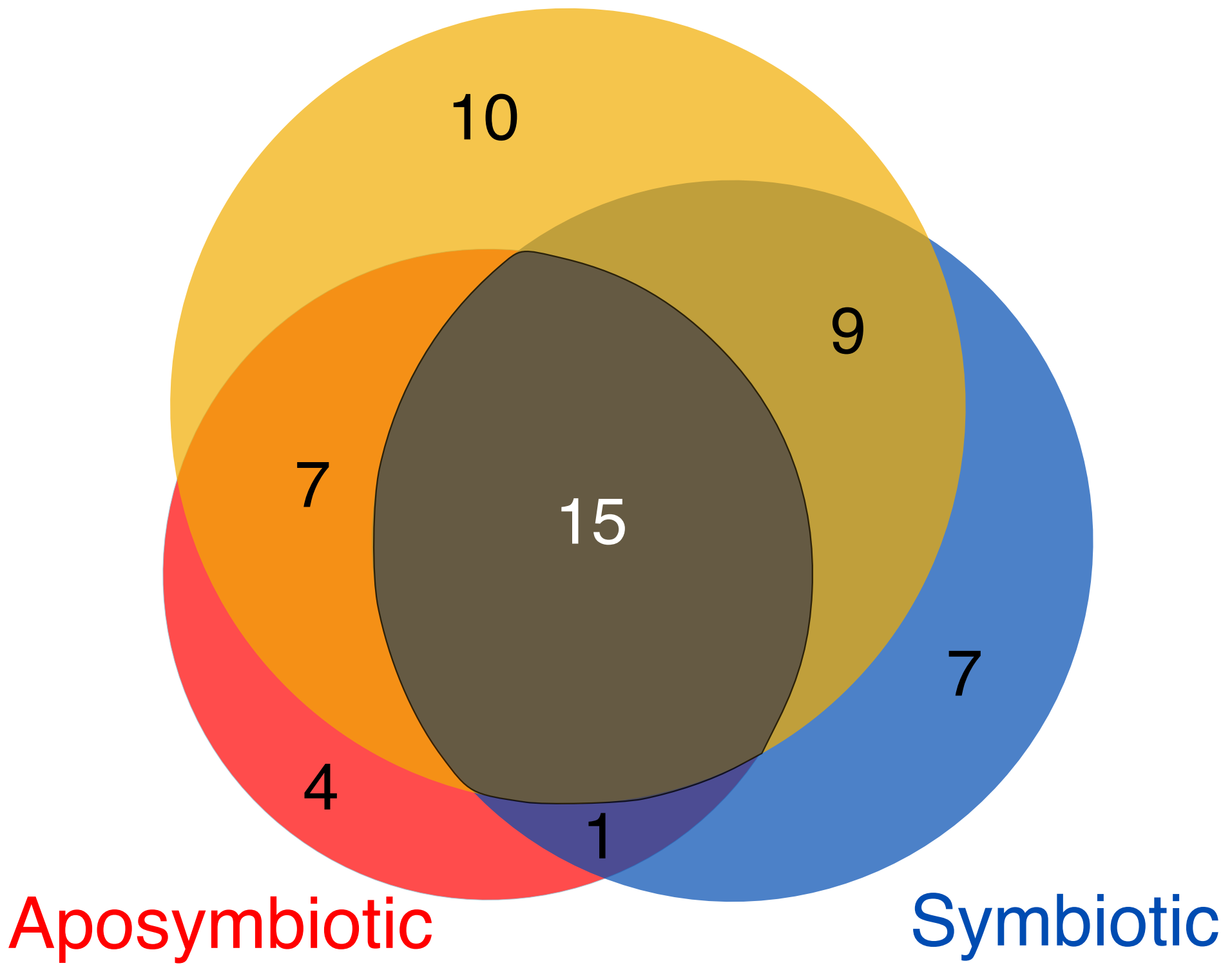

